Annales Academiæ Scientiarum Fennicæ

Mathematica

Volumen 38, 2013, 405-412

\title{
WHAT IS A CUBE?
}

\section{Tuomas Hytönen and Anna Kairema}

\author{
University of Helsinki, Department of Mathematics and Statistics \\ P.O. Box 68, FI-00014 University of Helsinki, Finland; tuomas.hytonen@helsinki.fi \\ University of Helsinki, Department of Mathematics and Statistics \\ P.O. Box 68, FI-00014 University of Helsinki, Finland; anna.kairema@helsinki.fi
}

\begin{abstract}
We give an intrinsic characterization of all subsets of a doubling metric space that can arise as a member of some system of dyadic cubes on the underlying space, as constructed by Christ.
\end{abstract}

\section{Introduction}

The notion of a cube in the usual Euclidean space does not need much explanation. Dyadic cubes are then certain special cubes with particular coordinate representations. The indispensable role of the dyadic cubes in Harmonic Analysis on Euclidean spaces has also motivated the construction of analogous nested structures in more general settings, most notably by Christ [2] in doubling metric spaces. However, this leads to a slight change in the point-of-view: there is no longer a notion of a 'cube' as such, and even a 'dyadic cube' barely makes sense as an individual object; it only becomes meaningful as a member of a system of dyadic cubes with useful intersection and covering properties reminiscent of those in the Euclidean case. Nevertheless, it is natural to ask the following question, which was posed to one of us by Bernicot:

What assumptions on a set do I have to put such that it can be considered one of the dyadic sets of a suitable dyadic system? [1]

In this note, we give a complete answer to this question, provided that a 'suitable dyadic system' is understood in the sense of the construction by Christ, which seems to be the most useful one at least for problems of singular integrals, and which we recall below. But let us first discuss the motivation to understand Bernicot's question.

First, many common arguments in Euclidean Harmonic Analysis involve the dyadic sub-cubes of a given (a priori, non-dyadic) cube. While it is quite clear what this means in the Euclidean space, the notion of a 'dyadic sub-cube' seems to become meaningless in an abstract space, unless we started from a dyadic cube from the beginning. Our characterization, however, provides an explicit way of testing whether a set qualifies as a dyadic cube. For such a set $E$, the existing techniques may be further pushed to yield a dyadic system $\mathscr{D}$ with $E \in \mathscr{D}$. After this, the dyadic sub-cubes of $E$ come as a part of the construction.

doi:10.5186/aasfm.2013.3838

2010 Mathematics Subject Classification: Primary 30L99; Secondary 51M05.

Key words: Dyadic cube, metric space, plumpness.

T.H. is supported by the European Research Council's Starting Grant "Analytic-probabilistic methods for borderline singular integrals", and by the Academy of Finland, grant 130166. Both authors are supported by the Academy of Finland, grant 133264. 
Another situation is the following: After the seminal work of Nazarov-TreilVolberg [10], it is now standard to treat singular integrals with respect to a nondoubling measure on $\mathbf{R}^{n}$ with the help of a random choice of the system of dyadic cubes. Since any cube of $\mathbf{R}^{n}$ can arise as a random dyadic cube in their construction, it is necessary to impose certain assumptions, such as the 'accretivity'

$$
\left|\frac{1}{\mu(Q)} \int_{Q} b \mathrm{~d} \mu\right| \geq \delta>0
$$

on the testing function $b$ in the $T b$ theorem, over the family of all cubes $Q \subset \mathbf{R}^{n}$. The $T b$ theorem of Nazarov-Treil-Volberg was generalized to the setting of an abstract metric space $X$ by Hytönen and Martikainen [5], but there it was left unclear, for which sets $Q \subset X$ exactly it is necessary to impose the above accretivity condition. The present characterization of all sets that can arise as dyadic cubes gives a clean form of this condition in the mentioned theorem.

The set-up for our characterization is the following. Let $(X, d)$ be a metric space. We assume that $X$ has the following (geometric) doubling property: There exists a positive integer $A_{1} \in \mathbf{N}$ such that for every $x \in X$ and $r>0$, the ball $B(x, r):=\{y \in X: d(y, x)<r\}$ can be covered by at most $A_{1}$ balls $B\left(x_{i}, r / 2\right)$. We call such metric spaces geometrically doubling.

To state our characterization, we formulate the following notion, which goes back to Martio-Väisälä [8] (a similar condition was used in [9]):

Definition 1.1. A set $E \subseteq X$ is plump with parameters $R>0$ and $b \in(0,1)$ if:

$$
\text { For all } y \in E \text { and } 0<r \leq R \text {, there exists } z \in E \text { such that }
$$

$$
B(z, b r) \subseteq B(y, r) \cap E .
$$

It turns out that a set $E$ can arise as a dyadic cube in $X$ if and only if both $E$ and $X \backslash E$ are plump, more precisely:

Theorem 1.3. Let $(X, d)$ be a geometrically doubling metric space. Given $E \subseteq$ $X$, the Christ-type dyadic cubes may be constructed in such a way that $\tilde{Q} \subseteq E \subseteq \overline{\bar{Q}}$, where $\tilde{Q}$ and $\bar{Q}$ are the interior and closure of some dyadic cube $Q$, if and only if $E$ is bounded and both $E$ and $X \backslash E$ are plump with parameters $b \in(0,1)$ (depending only on the space) and $R \gtrsim \operatorname{diam} E$.

We will provide a more precise quantitative formulation of the result in Proposition 3.1 and Proposition 3.3. The properties of dyadic cubes will be recalled in Section 2.1.

Acknowledgements. The authors thank an anonymous referee for very helpful comments on an earlier version of this paper.

\section{Definitions and lemmas}

We begin this section by recalling the dyadic structures. After that, we recall and study the notion of plumpness defined in the Introduction.

2.1. Dyadic cubes. In a geometrically doubling metric space $(X, d)$, a family of Borel sets $Q_{\alpha}^{k}, k \in \mathbf{Z}, \alpha \in I(k)$, is called a system of dyadic cubes with parameters 
$\delta \in(0,1)$ and $0<c_{1} \leq C_{1}<\infty$ if it has the following properties:

$$
X=\bigcup_{\alpha \in I(k)} Q_{\alpha}^{k} \quad \text { (disjoint union) } \forall k \in \mathbf{Z} \text {; }
$$

if $\ell \geq k$, then either $Q_{\beta}^{\ell} \subseteq Q_{\alpha}^{k}$ or $Q_{\alpha}^{k} \cap Q_{\beta}^{\ell}=\emptyset$;

$$
B\left(x_{\alpha}^{k}, c_{1} \delta^{k}\right) \subseteq Q_{\alpha}^{k} \subseteq B\left(x_{\alpha}^{k}, C_{1} \delta^{k}\right)=: B\left(Q_{\alpha}^{k}\right) ;
$$

if $\ell \geq k$ and $Q_{\beta}^{\ell} \subseteq Q_{\alpha}^{k}$, then $B\left(Q_{\beta}^{\ell}\right) \subseteq B\left(Q_{\alpha}^{k}\right)$.

The set $Q_{\alpha}^{k}$ is called a dyadic cube of generation $k$ with centre point $x_{\alpha}^{k} \in Q_{\alpha}^{k}$ and side length $\delta^{k}$. The interior and closure of $Q_{\alpha}^{k}$ are denoted by $\tilde{Q}_{\alpha}^{k}$ and $\bar{Q}_{\alpha}^{k}$, respectively. It follows from the geometric doubling property that the index set $I(k)$ is at most countable for each value of $k \in \mathbf{Z}$, and it can be assumed to be an initial interval in N.

Definition 2.6. We say that a set $\left\{x_{\alpha}^{k}\right\}_{k, \alpha} \subseteq X$ is a system of dyadic points with parameters $\delta \in(0,1)$ and $0<c_{0} \leq C_{0}<\infty$ if the following conditions are satisfied for every $k \in \mathbf{Z}$ :

$$
d\left(x_{\alpha}^{k}, x_{\beta}^{k}\right) \geq c_{0} \delta^{k} \quad(\alpha \neq \beta), \quad \min _{\alpha} d\left(x, x_{\alpha}^{k}\right)<C_{0} \delta^{k} \quad \forall x \in X .
$$

We say that a partial order $\leq$ among the index pairs $(k, \alpha)$ is a dyadic partial order for a given system of dyadic points, if it has the following properties:

- Every $(k+1, \beta)$ satisfies $(k+1, \beta) \leq(k, \alpha)$ for exactly one value of $\alpha$.

- For $\ell \leq k$, we have $(\ell, \beta) \leq(k, \alpha)$ if and only if $\ell=k$ and $\beta=\alpha$.

- For $\ell>k$, we have $(\ell, \beta) \leq(k, \alpha)$ if and only if there exist $\eta_{k}=\alpha, \eta_{k+1}, \ldots$, $\eta_{\ell-1}, \eta_{\ell}=\beta$ such that $\left(j+1, \eta_{j+1}\right) \leq\left(j, \eta_{j}\right)$ for every $j \in\{k, k+1, \ldots, \ell-1\}$.

- The relation $(k+1, \beta) \leq(k, \alpha)$ is almost determined by the proximity of the points in the sense of the two implications

$$
d\left(x_{\beta}^{k+1}, x_{\alpha}^{k}\right)<\frac{1}{2} c_{0} \delta^{k} \Rightarrow(k+1, \beta) \leq(k, \alpha) \Rightarrow d\left(x_{\beta}^{k+1}, x_{\alpha}^{k}\right)<C_{0} \delta^{k} .
$$

We recall from [4] the following result, which is a slight elaboration of seminal work by Christ [2]:

Theorem 2.8. Let $(X, d)$ be a geometrically doubling metric space. Suppose that there is a system of dyadic points $\left\{x_{\alpha}^{k}\right\}_{k, \alpha}$ with parameters $\delta \in(0,1)$ and $0<$ $c_{0} \leq C_{0}<\infty$ that satisfy $12 C_{0} \delta \leq c_{0}$, and a dyadic partial order $\leq$ among the index pairs $(k, \alpha)$. Then there exists a system of dyadic cubes $Q_{\alpha}^{k}$ with parameters $\delta, c_{1}=\frac{1}{3} c_{0}, C_{1}=2 C_{0}$, and centre points $x_{\alpha}^{k} \in Q_{\alpha}^{k}$. In fact, this system can be constructed in such a way that

$$
\tilde{Q}_{\alpha}^{k} \subseteq Q_{\alpha}^{k} \subseteq \bar{Q}_{\alpha}^{k}
$$

where

$$
\bar{Q}_{\alpha}^{k}=\overline{\left\{x_{\beta}^{\ell}:(\ell, \beta) \leq(k, \alpha)\right\}}
$$

and

$$
\tilde{Q}_{\alpha}^{k}=\operatorname{int} \bar{Q}_{\alpha}^{k}=\left(\bigcup_{\gamma \neq \alpha} \bar{Q}_{\gamma}^{k}\right)^{c}
$$


If either the system of points or the partial order is not given a priori, their existence already follows from the assumptions; however, we want to emphasize the fact that any given system of points and partial order can be used as a starting point.

Remark 2.12. The proof [4] shows that the second inclusion in (2.4) is true with $Q_{\alpha}^{k}$ replaced by $\bar{Q}_{\alpha}^{k}$.

2.13. Plumpness. We recall from the Introduction that a set $E \subseteq X$ is said to be plump with parameters $R>0$ and $b \in(0,1)$ if $E$ satisfies the following:

(2.14) For all $y \in E$ and $0<r \leq R$, there exists $z \in E: B(z, b r) \subseteq B(y, r) \cap E$.

Remark 2.15. 'Plumpness' has a close connection with other geometric notations. It is easily verified that in $\mathbf{R}^{n}$, being plump is equivalent to satisfying the corkscrew condition by Jerison-Kenig [6]: A domain $E$ in $\mathbf{R}^{n}$ is said to satisfy the interior (exterior) corkscrew condition if for some $R>0$ and $b \in(0,1)$, and every boundary point $y \in \partial E$ and $0<r<R$, there exists $z \in B(y, r) \cap E$ $\left(z \in B(y, r) \cap\left(\mathbf{R}^{n} \backslash \bar{E}\right)\right)$ such that $\operatorname{dist}(z, \partial E) \geq b r$.

Plumpness also resembles the notion of porosity which goes back at least to Denjoy [3, pp. 195-196] and has been investigated widely by several authors. A set $E$ in $\mathbf{R}^{n}$ is uniformly lower porous if for some $b \in(0,1)$ and for every $y \in E$ and $0<r<\operatorname{diam}(E)$, the set $B(y, r) \cap\left(\mathbf{R}^{n} \backslash E\right)$ contains a ball $B(z, b r)$. These two notions are, however, not the same. For example, any ball $B(x, R)$ in $\mathbf{R}^{n}$ and its complement are plump but neither one is porous.

Examples 2.16. 1. Examples of plump sets in $\mathbf{R}^{n}$ are provided by John domains, first introduced by John [7]: A domain $\Omega$ in $\mathbf{R}^{n}$ is $(\alpha, \beta)$-John domain if there exists a point $x_{0} \in \Omega$ ('central point') such that given any $x \in \Omega$, there exists a rectifiable path $\gamma:[0, \ell] \rightarrow \Omega$ which is parametrized by arclength, such that $\gamma(0)=x, \gamma(\ell)=x_{0}, \ell \leq \beta$ and

$$
\operatorname{dist}(\gamma(t), \partial \Omega) \geq \frac{\alpha}{\ell} t \quad \forall t \in[0, \ell] .
$$

Every John domain satisfies the corkscrew condition [9, Lemma 6.3] and thus, is a plump set.

2. The well established non-tangentially accessible domains (NTA domains), introduced by Jerison-Kenig [6], satisfy both the interior and exterior corkscrew condition by definition. Thus, by the main result of the present paper, every bounded NTA domain in $\mathbf{R}^{n}$ qualifies as a dyadic cube.

We record the following easy observation.

Lemma 2.17. Suppose $E$ is plump with parameters $R>0$ and $b \in(0,1)$. Then

(1) $E$ is plump with any parameters $\tilde{R} \leq R$ and $0<\tilde{b} \leq b$;

(2) $E$ is plump with any parameters $\tilde{R} \geq R$ and $\tilde{b} \in(0,1)$ that satisfy $\tilde{R} \tilde{b} \leq R b$.

Proof. The first assertion is obvious. For the second assertion, let $\tilde{R} \geq R$ and $\tilde{b} \in(0,1)$ be such that $\tilde{R} \tilde{b} \leq R b$. Suppose $y \in E$ and $0<r \leq \tilde{R}$. Then $0<r R / \tilde{R}=: t \leq R$ so that there exists $z \in E$ such that

$$
B(z, \tilde{b} r)=B\left(z, \frac{\tilde{b} \tilde{R}}{R} t\right) \subseteq B(z, b t) \subseteq B(y, t) \cap E \subseteq B(y, r) \cap E .
$$


We give next a definition for a dyadically plump set, that better suits our purposes.

Definition 2.18. A set $E \subseteq X$ is dyadically plump (d-plump) with parameters $\delta \in(0,1), m \in \mathbf{Z}$ and $0<b_{0} \leq B_{0}<\infty$ if $E$ satisfies the following:

(2.19) For all $y \in E$ and $k \geq m$, there exists $z \in E: B\left(z, b_{0} \delta^{k}\right) \subseteq B\left(y, B_{0} \delta^{k}\right) \cap E$.

Qualitatively, set $E$ is plump if and only if $E$ is d-plump. Quantitatively, the relationship is formulated in the following lemma.

Lemma 2.20. If $E$ is a plump set with parameters $R>0$ and $b \in(0,1)$, then $E$ is d-plump with any parameters $\delta \in(0,1), m \in \mathbf{Z}$ and $0<b_{0} \leq B_{0}<\infty$ that satisfy $b_{0} / B_{0} \leq b$ and $B_{0} \delta^{m} \leq R$. Conversely, if $E$ is a d-plump set with parameters $\delta \in(0,1), m \in \mathbf{Z}$ and $0<b_{0} \leq B_{0}<\infty$, then $E$ is plump with any parameters $R>0$ and $b \in(0,1)$ that satisfy $b \leq \delta b_{0} / B_{0}$ and $R \leq B_{0} \delta^{m-1}$.

Proof. For the first assertion, let $\delta \in(0,1)$, and suppose that $0<b_{0} \leq B_{0}<\infty$ are such that $b_{0} / B_{0} \leq b$. Then pick $m \in \mathbf{Z}$ that satisfies $B_{0} \delta^{m} \leq R$. Let $y \in E$ and $k \geq m$. Then, by (2.14) with $r=B_{0} \delta^{k} \leq B_{0} \delta^{m} \leq R$, there exists $z \in E$ such that

$$
B\left(z, b_{0} \delta^{k}\right) \subseteq B(z, b r) \subseteq B(y, r) \cap E=B\left(y, B_{0} \delta^{k}\right) \cap E,
$$

which shows that $E$ is d-plump.

For the second assertion, suppose that $b \in(0,1)$ and $R>0$ are such that $b \leq \delta b_{0} / B_{0}$ and $R \leq B_{0} \delta^{m-1}$. Let $y \in E$ and $0<r \leq R$, and let $k \geq m$ be an integer that satisfies $B_{0} \delta^{k}<r \leq B_{0} \delta^{k-1}$. Then, by (2.19), there exists $z \in E$ such that

$$
B(z, b r) \subseteq B\left(z, b_{0} \delta^{k}\right) \subseteq B\left(y, B_{0} \delta^{k}\right) \cap E \subseteq B(y, r) \cap E .
$$

\section{The proof of the main result}

In this section we will provide a proof for the quantitative version of our main result, Theorem 1.3, which is formulated in Propositions 3.1 and 3.3 below.

Proposition 3.1. Suppose that $\mathscr{D}$ is a family of dyadic cubes with parameters $\delta \in(0,1)$ and $0<c_{1} \leq C_{1}<\infty$. Let $Q_{\alpha}^{m} \in \mathscr{D}$ be a dyadic cube of side length $\delta^{m}$. Then both $F \in\left\{Q_{\alpha}^{m}, X \backslash Q_{\alpha}^{m}\right\}$ are d-plump with parameters $\delta, m, b_{0}=c_{1}$ and $B_{0}=c_{1}+C_{1}$. In particular, $Q_{\alpha}^{m}$ is plump with parameters $b=\delta c_{1} /\left(c_{1}+C_{1}\right)$ and

$$
R=\frac{c_{1}+C_{1}}{2 \delta C_{1}} \operatorname{diam}\left(Q_{\alpha}^{m}\right)
$$

Proof. It suffices to consider the case $F=Q_{\alpha}^{m}$ and $y \in Q_{\alpha}^{m}$ since otherwise, $y \in Q_{\beta}^{m}$ for some $\beta \neq \alpha$, and we argue similarly with $\alpha$ replaced by $\beta$.

To this end, suppose $y \in Q_{\alpha}^{m}$ and $k \geq m$. By (2.2) and (2.3), $y \in Q_{\beta}^{k}$ for some $Q_{\beta}^{k} \subseteq Q_{\alpha}^{m}$. Thus, by (2.4),

$$
d\left(y, x_{\beta}^{k}\right)<C_{1} \delta^{k}
$$

We will show that $z=x_{\beta}^{k}$ satisfies (2.19). First note that $B\left(x_{\beta}^{k}, c_{1} \delta^{k}\right) \subseteq Q_{\beta}^{k} \subseteq Q_{\alpha}^{m}$. We are left to show that $B\left(x_{\beta}^{k}, c_{1} \delta^{k}\right) \subseteq B\left(y, B_{0} \delta^{k}\right)$. To this end, suppose $x \in B\left(x_{\beta}^{k}, c_{1} \delta^{k}\right)$ and note that, by (3.2),

$$
d(x, y) \leq d\left(x, x_{\beta}^{k}\right)+d\left(x_{\beta}^{k}, y\right) \leq c_{1} \delta^{k}+C_{1} \delta^{k}=B_{0} \delta^{k} .
$$


This shows that $E$ is d-plump with parameters $\delta, m, b_{0}=c_{1}$ and $B_{0}=c_{1}+C_{1}$. By Lemma 2.20, $Q_{\alpha}^{m}$ is plump and we may choose $b=\delta b_{0} / B_{0}=\delta c_{1} /\left(c_{1}+C_{1}\right)$ and $R=B_{0} \delta^{m-1}=\left(c_{1}+C_{1}\right) \delta^{m-1}$. Since $\operatorname{diam}\left(Q_{\alpha}^{m}\right) \leq 2 C_{1} \delta^{m}$ by $(2.4)$, the proof is completed by Lemma 2.17(1).

Proposition 3.3. Let $E \subseteq X$, and suppose both $F \in\{E, X \backslash E\}$ are d-plump with parameters $\delta \in(0,1), m \in \mathbf{Z}$ and $0<b_{0} \leq B_{0}<\infty$ where diam $E \leq B_{0} \delta^{m}$ and $12 B_{0} \delta \leq b_{0}$. Then the Christ-type dyadic cubes may be constructed in such a way that $E$ arises as a dyadic cube. More precisely, there exists a dyadic system $\mathscr{D}$ with parameters $\delta, c_{1}=b_{0} / 3$ and $C_{1}=2 B_{0}$, and a dyadic cube $Q \in \mathscr{D}$ of side length $\delta^{m}$ such that $\tilde{Q} \subseteq E \subseteq \bar{Q}$.

The proof of Proposition 3.3 consists of three lemmata.

Lemma 3.4. (Choice of dyadic points) Under the assumptions and with the fixed values of parameters as in Proposition 3.3, let $F \in\{E, X \backslash E\}$. Then for every $k \in \mathbf{Z}$, there exists a set $\left\{x_{\alpha}^{k}\right\}_{\alpha}$ of points with the following properties:

$$
d\left(x_{\alpha}^{k}, x_{\beta}^{k}\right) \geq b_{0} \delta^{k} \quad(\alpha \neq \beta), \quad \min _{\alpha} d\left(x, x_{\alpha}^{k}\right)<B_{0} \delta^{k} \quad \forall x \in X ;
$$

If $k \geq m$, then moreover

$$
\min _{\alpha: x_{\alpha}^{k} \in F} d\left(x, x_{\alpha}^{k}\right)<B_{0} \delta^{k} \quad \forall x \in F \in\{E, X \backslash E\}
$$

and

$$
\operatorname{dist}\left(x_{\alpha}^{k}, X \backslash F\right) \geq b_{0} \delta^{k} \quad \forall x_{\alpha}^{k} \in F .
$$

If $k=m$, then there is exactly one $\alpha$ such that $x_{\alpha}^{m} \in E$.

Proof. We first observe that

$$
\left\{x \in F: d(x, X \backslash F) \geq b_{0} \delta^{m}\right\} \neq \emptyset
$$

for both choices of $F$. To this end, pick a $y \in F$. We apply (2.19) with $k=m$, and find a point $z \in F$ such that

$$
B\left(z, b_{0} \delta^{m}\right) \subseteq F \cap B\left(y, B_{0} \delta^{m}\right)
$$

and thus $d(z, X \backslash F) \geq b_{0} \delta^{m}$.

For $k \geq m$ and both choices of $F$, we pick a maximal set $\left\{x_{\alpha}^{k}\right\}_{\alpha}$, of points in $F$ that satisfies the two conditions

$$
d\left(x_{\alpha}^{k}, x_{\beta}^{k}\right) \geq b_{0} \delta^{k} \quad(\alpha \neq \beta) \quad \text { and } \quad \operatorname{dist}\left(x_{\alpha}^{k}, X \backslash F\right) \geq b_{0} \delta^{k} .
$$

By (3.8), both these collections are nonempty. We equip them with individual labels to form one joint collection $\left\{x_{\alpha}^{k}\right\}_{\alpha}$. It still satisfies

$$
d\left(x_{\alpha}^{k}, x_{\beta}^{k}\right) \geq b_{0} \delta^{k} \quad(\alpha \neq \beta):
$$

if both $x_{\alpha}^{k}, x_{\beta}^{k}$ belong to the same $F \in\{E, X \backslash E\}$, this is part of the construction, and if $x_{\alpha}^{k} \in F, x_{\beta}^{k} \in X \backslash F$, then

$$
d\left(x_{\alpha}^{k}, x_{\beta}^{k}\right) \geq d\left(x_{\alpha}^{k}, X \backslash F\right) \geq b_{0} \delta^{k} \quad(\alpha \neq \beta) .
$$

For $k=m$, we throw away all but one $x_{\alpha}^{m} \in E$, which we denote by $x_{\alpha_{0}}^{m}$.

We need to check that the points $x_{\alpha}^{k}$ are $B_{0} \delta^{k}$-dense in $F$ for both choices of $F$. If $F=E$ and $k=m$, then all $x \in E$ satisfy $d\left(x, x_{\alpha_{0}}^{m}\right) \leq \operatorname{diam} E<B_{0} \delta^{m}$. Let then either $k>m$ or $k=m$ and $F=X \backslash E$, and consider an arbitrary $x \in F$. We apply 
(2.19), and find a point $z \in F$ such that $B\left(z, b_{0} \delta^{m}\right) \subseteq F \cap B\left(x, B_{0} \delta^{m}\right)$. In particular, $\operatorname{dist}(z, X \backslash F) \geq b_{0} \delta^{m}$. If also $d\left(z, x_{\alpha}^{m}\right) \geq b_{0} \delta^{m}$ for all $x_{\alpha}^{m} \in F$, then $z$ could have been added to the collection $\left\{x_{\alpha}^{m}\right\}_{\alpha}$, contradicting its maximality. Thus, there exists $x_{\alpha}^{m} \in B\left(z, b_{0} \delta^{m}\right) \subseteq F \cap B\left(x, B_{0} \delta^{m}\right)$; i.e., $x_{\alpha}^{m} \in F$ and $d\left(x, x_{\alpha}^{m}\right)<B_{0} \delta^{m}$, which is as claimed in (3.6).

Finally, for $k<m$, we pick a maximal set $\left\{x_{\alpha}^{k}\right\}_{\alpha}$, of points in $X$ that satisfy the first condition in (3.5), and then by maximality also the second condition in (3.5), since $B_{0} \geq b_{0}$. For $k>m$, there are no further conditions required, so we are done.

Note that the point set $\left\{x_{\alpha}^{k}\right\}_{k, \alpha}$ provided by Lemma 3.4 is, in particular, a system of dyadic centre points with parameters $\delta$ and $0<b_{0} \leq B_{0}<\infty$ that satisfy $12 B_{0} \delta \leq b_{0}$.

The next step in the construction of dyadic cubes is the choice of a partial order for the dyadic index pairs $(k, \alpha)$, which will describe the cubes' child-parent (descendant-ancestor) relationships.

Lemma 3.9. (Choice of dyadic partial order) Under the assumptions and with the fixed values of parameters as in Proposition 3.3, there is a dyadic partial order $\leq$ among the pairs $(k, \alpha)$ with the following additional property:

If $k \geq m$ and $(\ell, \beta) \leq(k, \alpha)$, then $x_{\beta}^{\ell}$ and $x_{\alpha}^{k}$ belong to the same set $F \in\{E, X \backslash E\}$.

Proof. We define a partial order as follows. Given $k \geq m$ and a point $x_{\beta}^{k+1} \in F$, check whether there exists $\alpha$ such that $d\left(x_{\alpha}^{k}, x_{\beta}^{k+1}\right)<b_{0} \delta^{k} / 2$. If one exists, it is necessarily unique by (3.5), and moreover, $x_{\alpha}^{k} \in F$ by (3.7). We then decree that $(k+1, \beta) \leq(k, \alpha)$. If no such good $\alpha$ exists, choose any $\alpha$ for which $x_{\alpha}^{k} \in F$ and $d\left(x_{\alpha}^{k}, x_{\beta}^{k+1}\right)<B_{0} \delta^{k}$, and decree that $(k+1, \beta) \leq(k, \alpha)$; at least one such $\alpha$ exists by (3.6). In either case, we decree that $(k+1, \beta)$ is not related to any other $(k, \gamma)$. Note that the additional above property is clear from this construction.

Given $k<m$ and a point $(k+1, \beta)$, we proceed in the same way as before except that we drop the requirement $x_{\alpha}^{k} \in F$. Finally, we extend $\leq$ by transitivity to obtain a partial ordering.

With the dyadic points and the partial order at hand, Theorem 2.8 guarantees the existence of a system of dyadic cubes $Q_{\alpha}^{k}$ with parameters $\delta, c_{1}=\frac{1}{3} b_{0}$ and $C_{1}=2 B_{0}$. The proof of Proposition 3.3 is now completed by the following lemma.

Lemma 3.10. If $\alpha_{0}$ is the unique index with $x_{\alpha_{0}}^{m} \in E$, we have $\tilde{Q}_{\alpha_{0}}^{m} \subseteq E \subseteq \bar{Q}_{\alpha_{0}}^{m}$.

Proof. Suppose $x \in E$. Then, by (3.6), for every $k \geq m$ there exists $x_{\beta}^{k} \in E$ such that $d\left(x_{\beta}^{k}, x\right)<B_{0} \delta^{k} \rightarrow 0$ as $k \rightarrow \infty$. This shows that

$$
x \in \overline{\left\{x_{\beta}^{k}:(k, \beta) \leq\left(m, \alpha_{0}\right)\right\}}=\bar{Q}_{\alpha_{0}}^{m} .
$$

To show that $\tilde{Q}_{\alpha_{0}}^{m} \subseteq E$, it suffices to show that

$$
X \backslash E \subseteq\left(\tilde{Q}_{\alpha_{0}}^{m}\right)^{c}=\bigcup_{\alpha \neq \alpha_{0}} \bar{Q}_{\alpha}^{m} .
$$

To this end, suppose $x \in X \backslash E$. Then, by (3.6), for every $k \geq m$ there exists $x_{\beta}^{k} \in X \backslash E$ such that $d\left(x_{\beta}^{k}, x\right)<B_{0} \delta^{k} \rightarrow 0$ as $k \rightarrow \infty$. By Lemma 3.9, for each such 
$k$ we have that $(k, \beta) \leq(m, \alpha)$ with some $x_{\alpha}^{m} \in X \backslash E$. This implies that $x_{\beta}^{k} \in \bar{Q}_{\alpha}^{m}$ for some $\alpha \neq \alpha_{0}$, and hence $d\left(x_{\beta}^{k}, x_{\alpha}^{m}\right)<2 B_{0} \delta^{m}$ by (2.4); see also Remark 2.12. For all such $\alpha$, we have $d\left(x_{\alpha}^{m}, x\right) \leq d\left(x_{\alpha}^{m}, x_{\beta}^{k}\right)+d\left(x_{\beta}^{k}, x\right)<2 B_{0} \delta^{m}+B_{0} \delta^{k} \leq 3 B_{0} \delta^{m}$, and hence geometric doubling implies that there are only boundedly many relevant $x_{\alpha}^{m}$ here. Passing to a subsequence over $k$, we may assume that all $x_{\beta}^{k}$ belong to the same $\bar{Q}_{\alpha_{1}}^{m}$ with $\alpha_{1} \neq \alpha_{0}$. Thus also

$$
x=\lim _{k \rightarrow \infty} x_{\beta}^{k} \in \bar{Q}_{\alpha_{1}}^{m} \subseteq \bigcup_{\alpha \neq \alpha_{0}} \bar{Q}_{\alpha_{0}}^{m} .
$$

This completes the proof of Proposition 3.3, and thereby also the proof of Theorem 1.3.

\section{References}

[1] Bernicot, F.: Personal communication, 2011.

[2] Christ, M.: A $T(b)$ theorem with remarks on analytic capacity and the Cauchy integral. Colloq. Math. 60/61:2, 1990, 601-628.

[3] Denjoy, A.: Leçons sur le Calcul des Coefficients d'une Série Trigonométrique. Part II. Gauthier-Villars, 1941.

[4] Hytönen, T., and A. Kairema: Systems of dyadic cubes in a doubling metric space. - Colloq. Math. 126:1, 2012, 1-33.

[5] Hytönen, T., and H. Martikainen: Non-homogeneous Tb theorem and random dyadic cubes on metric measure spaces. - J. Geom. Anal. 22:4, 2012, 1071-1107.

[6] Jerison, D.S., and C.E. Kenig: Boundary behaviour of harmonic functions in nontangentially accessible domains. - Adv. Math. 46:1, 1982, 80-147.

[7] John, F.: Rotation and strain. - Comm. Pure Appl. Math. 14:3, 1961, 391-413.

[8] Martio, O., and J. VÄIsÄLÄ: Global $L^{p}$-integrability of the derivatives of a quasiconformal mapping. - Complex Var. Theory Appl. 9:4, 1988, 309-319.

[9] Martio, O., and M. Vuorinen: Whitney cubes, p-capacity and Minkowski content. - Expo. Math. 5, 1987, 17-40.

[10] Nazarov, F., S. Treil, and A. Volberg: The Tb-theorem on non-homogeneous spaces. Acta Math. 190:2, 2003, 151-239.

Received 20 September 2012 • Accepted 19 February 2013 